\title{
CVS: fast cardinality estimation for large-scale data streams over sliding windows
}

\author{
Jingsong Shan ${ }^{\mathrm{a}, \mathrm{b}}$, Jianxin Luo ${ }^{\mathrm{a}}$, Guiqiang $\mathrm{Ni}^{\mathrm{a}, *}$, Zhaofeng $\mathrm{Wu}^{\mathrm{a}}$, Weiwei Duan ${ }^{\mathrm{a}}$ \\ ${ }^{a}$ College of Command Information Systems, PLA University of Science and Technology, \\ Nanjing, China \\ ${ }^{b}$ The Faculty of Computer Engineering,Huaiyin Institute of Technology, Huaian, China
}

\begin{abstract}
Estimating the cardinality of data streams over a sliding window is an important problem in many applications, such as network traffic monitoring, web access log analysis and database. The problem becomes more difficult in largescale data streams when time and space complexity is taken into account. In this paper, we present a novel randomized data structure to address the problem. The significant contributions are as follows. (1) A space-efficient counter vector sketch (CVS) are proposed, which extends the well-known bitmap sketch to sliding window settings. (2) Based on the CVS, a random update mechanism is introduced, whereby a small fixed number of entries are randomly chosen from CVS in a step and then updated. This means that the update procedure just costs constant time. (3) Furthermore, estimating cardinality by CVS just needs one-pass scan of the data. (4) Finally, a theoretical analysis is given to show the accuracy of CVS-based estimators. Our comprehensive experiments confirm that the CVS-based schema attains high accuracy, and that its time efficiency in comparison with the timestemp vector (TSV) and the auxiliary indexing method.
\end{abstract}

Keywords: Data stream, Sliding window, Counter vector sketch, Cardinality, Random update

\footnotetext{
* Corresponding author

Email addresses: shanjings@hyit.edu.cn (Jingsong Shan), ljx555@163.com (Jianxin Luo), networkmanage707@gmail.com (Guiqiang Ni), qqk20080915@163.com (Zhaofeng Wu), duanweiwei@vip.163.com (Weiwei Duan)
}

Preprint submitted to Neurocomputing

December 1, 2015

(C) 2016. This manuscript version is made available under the Elsevier user license http://www.elsevier.com/open-access/userlicense/1.0/ 


\section{Introduction}

Massive data analysis is often performed in a streaming manner, where data are continuously generated at a high rate in a form of data streams [1, 2, 3]. Processing data streams attracts much attention in recent years because of requirements in real-time big data analysis, such as neural network based methods 4, 5, 6, 7, classification based approaches 8, 9, 10. In this paper, we focus on an important aspect in data stream processing: estimating cardinality for large-scale data streams over sliding windows. The cardinality of a data stream $S$ is the number of distinct items in $S$. Counting the cardinality of a data stream plays an important role in many applications, such as database query [2], computer network monitoring [11, 12], anomaly detection [13].

In many real world applications, such as traffic engineering, web log analysis, data streams are of unbounded length. The quantity of data collected is too huge to fit in main memory. Given memory constrains, it is necessary to devise techniques for maintaining a summary of history data items. Since 1980s, a variety of space-efficient randomized data structures (or sketches) 14 have been developed for estimating cardinality of a data stream, such as Probabilistic Counting [15, Bitmap Sketch [16, Min-count [17, LogLog counting [18]. Similar to Bloom Filter [19], these sketches consume much smaller memory space than the actual data set because they only maintain a concise summary of the data set. These sketches work well on static data streams, where all data observed so far are involved in. However, in most applications, very old data items are considered less useful and relevant than the more recent data. Thus stale data items should be discarded over time from these sketch structures. A commonly-used approach for discounting outdated items is the sliding window model [20]. Data streams over sliding windows are dynamic, where data items can be added or deleted dynamically as time goes by.

Sketches [16, 15, 18, 17, mentioned above cannot work directly in such dynamical settings. Firstly, these sketches have no timing schema by which stale 
information can be evicted out. Secondly and importantly, the random mapping mechanism completely destroys the temporal correlation between cells of a sketch. This means that these cells are temporally unordered. It is difficult to timely evict expired information from the temporally unordered sketch based on a sliding window.

Few previous works address the problem of adapting sketches to sliding window model. Hyang-Ah Kim et al. extend bitmap sketch [16] to TimeStamp Vector (TSV) 21 sketch. Its basic idea is to replace a bit in bitmap sketch with a real timestamp. All cells of the TSV must be probed and checked for discarding the aged information, a very time-consuming process for a large window size. It is infeasible for real-time online applications. To speed up the process, some researchers [22, 23] introduce an indexed sketch to rearrange the cells in time order. However, the auxiliary indexing structure requires too large memory to be used in massive data streams.

In this paper, we present a counter vector sketch (CVS) for estimating the cardinality of data streams over a sliding window. The key difference between CVS and other window-based sketches [21, 22] is that the eviction of stale information in CVS only needs to randomly select few of all sketch cells, instead of the whole. The CVS runs much faster and needs less memory space than TSV 21] and the indexed sketch [22, 23].

The contributions of this paper are as follows:

- We propose a space-time efficient counter vector sketch (CVS), a novel randomized data structure which extends the bitmap sketch [16] to sliding window settings.

- Based on the CVS, a random update mechanism is introduced for timely evicting expired information from the CVS, which only costs $O(1)$ time (constant time) in each step.

- A CVS-based algorithm is provided to estimate the cardinality of data streams, taking only one pass of the data. 
- A theoretical analysis is provided to guarantee the accuracy of CVS-based estimators. Our comprehensive experiments confirm that the CVS-based schema attains high accuracy, and demonstrate the time efficiency of the CVS.

The rest of this paper is organized as follows. Section 2 describes the problem statement and related preliminaries. Section 3 introduces our proposed counter vector sketch and provides a theoretical error analysis. Experimental results are reported in Section 4 , followed by conclusion and discussion in Section 5 .

\section{Preliminaries}

\subsection{Problem Statement}

A data stream $S=\left\{e_{1}, e_{2}, \cdots, e_{i}, \cdots\right\}$ is seen as a sequence of records, where a record may be composed of numbers, strings, or other types. The data stream size $N$ is potentially infinite.

This paper is to solve the following problem: Given a sliding window of size $W$ and a data stream $S$, estimate the cardinality of the data stream over the sidling window.

Because the key space in a data stream may be extremely huge, e.g., key space of network flows can be as much as $2^{100}$, and the size of sliding windows $W$ may be very large (as much as billions of items). Thus, in most large-scale data stream settings, computing the precise cardinality over sliding windows is too expensive in space and time to be practically feasible. In this paper, we propose a novel space-time efficient sketch to approximate the cardinality of data streams.

Because the bitmap sketch is a building block of our approach, we review it briefly.

\subsection{Bitmap sketch}

Difinition 1. (Bitmap Sketch) A bitmap sketch is defined as a bit vector of size $m, V[m]$, which only stores a summary of the data stream and requires substantively smaller memory space than the actual data. 


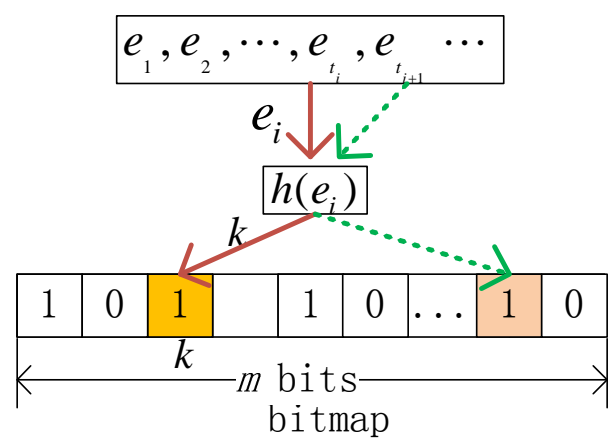

Figure 1: Typical structure of a bitmap sketch

A typical structure of a bitmap sketch is shown in Fig. 11. The bitmap sketch only maintains a bit vector of size $m$, much smaller than the actual data set. As has been demonstrated by Metwally et al. in 24] and K. Aouiche et al. in [25], bitmap sketch has impressive advantages over others (Probabilistic Counting [15], Min-count [17], LogLog counting [18]). For the bitmap sketch to attain comparable accuracy, it needs less memory space than other sketches.

The bitmap sketch supports two operations: setting and query. For each element $e$, a uniformly distributed hash function $h(x)$ is used to map $e$ to a bit position $j$. The corresponding bit at position $j$ is set to 1 . The process is called insertion operation or setting operation. After $N$ iterations, each bit of the vector has one of the two states: empty (or 0) or non-empty (or 1). By the ratio of the zero number to size $m$, one can estimate the approximate cardinality of a data set seen so far. The estimation is called a query process. The estimating formula is shown in Equation 1.

$$
\hat{n}=-m \ln \frac{z}{m}
$$

where $m$ is the size of the bitmap, $z$ is the number of empty cells. The estimation accuracy increase with the size of the bitmap. Further details can be found in [16] 24]. 


\subsection{Difficulties of bitmap sketch under sliding window scenarios}

In practical applications, it is often necessary to maintain statistics with regard to the last $W$ data items of a data stream. This results in the sliding window model [20, 26]: each data item expires after exactly $W$ time steps. In this model, data items are added into or deleted from a window over time, dynamically.

The bitmap sketch is challenged in such dynamical setting, given time and memory space are constrained. Firstly, bitmap sketch has to be extended to support the timing mechanism by which the expired information can be forgotten over time. And, to accommodate massive data streams, this extension allows only a very small amount of memory space.

The biggest challenge for bitmap sketch is to timely evict outdated information from the window over time. The entries of bitmap sketch are temporally unordered because of randomly mapping mechanism. To obtain precise results, there exit two choices: traverse all cells and check if they are expired (too slow) [21], or sort all cells in time order by auxiliary indexing (too much memory needed) 22] 23.

Additionally, algorithms for data streams often work in online settings with a single-pass through the data. For example, on an Internet backbone link packets arrive at a rate of tens of Gigabit per second. The time interval between two neighboring packets is as small as tens of nanoseconds. In this case, it is impossible to revisit old data items for counting due to the extremely high speed.

\section{Our solution: counter vector sketch}

This section first introduces the proposed counter vector sketch, and then explains the random update mechanism. Next, a theoretical analysis is provided to guarantee the accuracy of CVS-based estimators. Lastly, this section studies the tradeoff between accuracy, memory and time. 


\subsection{Counter vector sketch}

Difinition 2. A Counter Vector Sketch (CVS) is defined as a vector of counters $V[1] ; \cdots ; V[m]$, each of which has minimum value 0 and maximum value $C$. To clearly differentiate bits in a bitmap sketch, each counter of a CVS is called an entry.

The proposed CVS is an extension of bitmap sketch. Unlike the bitmap sketch, CVS is able to indicate the aging degree of an entry and evict outdated information from sliding windows; an entry of CVS is an integer (or a counter) but a cell of bitmap sketch is a bit. Besides this, CVS also remarkably decreases the memory requirements compared with TSV [21] and the indexed sketch [22]. This is because that each entry of CVS only needs to maintain a small integer, whereas a 64-bit timestamp in TSV and additional indexing in the indexed sketch. This issue will further be discussed in subsection 4.4 .

The purpose of changing a bit in bitmap sketch into a counter in CVS is to denote the aging degree of an entry. If an entry $V[i]=C$, the entry is the newest. If an entry $V[i]=0$, the entry is expired and should be evicted out of the sliding window. The CVS supports following three operations: setting, query and deletion operation. Details of the algorithm is shown in Table 1 . The notations throughout the paper is listed in Table 2 .

The setting (or insertion) operation is very similar to bitmap sketch. At the beginning, each entry is initialized to 0. If a newly arrived item is mapped to the entry $i$ by an uniform hash function $h(x), V[i]$ is set to the maximum value $C$. The CVS has the same query process as a bitmap sketch. All we need to do is to estimate the approximate cardinality of data set using Equation 1 . The deletion operation will be discussed in the following subsection.

\subsection{Random update mechanism}

In this paper, we employ a random update mechanism to discard the aged information from CVS. Its definition is shown in Definition 3. The procedure is used to maintain CVS, shown in Algorithm 1. 
Table 1: Algorithm for maintaining and updating CVS over a sliding window

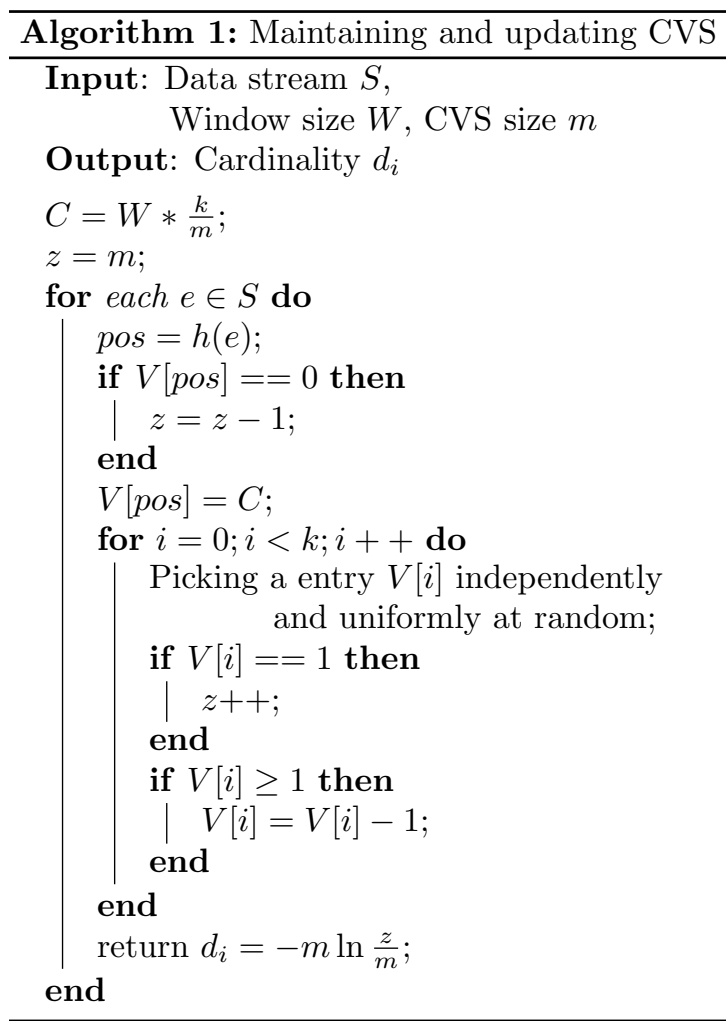

Table 2: Notations and its meaning

\begin{tabular}{cl}
\hline Notations & Description \\
\hline$S$ & Data stream \\
$W$ & Size of a sliding window \\
$m$ & Size of CVS \\
$h$ & A uniform hash function which maps $e \in S$ to $[0,1, \cdots, m-1]$ \\
$C$ & Maximum value a entry is set to \\
$k$ & Number of entries chosen to be decremented by 1 in each step \\
$K_{w}$ & Number of times an entry is picked in W steps \\
$p$ & Probability that a entry is chosen in each step \\
$z$ & Number of empty entry in the CVS \\
\hline
\end{tabular}


Difinition 3. A random update mechanism is a way of evicting the obsolete information from CVS. Specifically, in each step, $k(k \geq 1)$ entries are chosen independently at random, each of which is decremented by one if its value is greater than 0.

The striking advantage of the approach is that the deletion operation only requires constant running time in each step. A similar method is presented in 27. which focuses on detecting duplicates based on a bloom filter.

\subsection{Configuring the maximum value $C$ of a counter}

Given the sliding window size $W$ and CVS size $m$, a key problem we should address is to calculate the parameter $C$ such that CVS satisfies the following eviction rule over sliding windows:

Suppose that an entry $i$ is set to the maximum $C$ at time $t$ and no elements are mapped to the entry in the following $W$ steps (or, equivalently, a time interval spanning a sliding window), the entry should be decremented to be 0 (i.e., the information in $V[i]$ is evicted from the sliding window due to expiry) with high probability.

To set parameter $C$, we first introduce the following theorem.

Theorem 1. Given a CVS with $m$ entries, a sliding window of size $W$, if $k$ entries are picked uniformly and independently at random to be decremented by 1 in each step, the expected number of times an entry is picked is $\frac{W * k}{m}$.

proof: Let Indicator Variable $I_{i}=1$ if $V[i]$ is chosen in step $i$. Otherwise $I_{i}=0$. Because each pick is independent and uniform in a step. We obtain: $\operatorname{Pr}\left(I_{i}=1\right)=k / m$. Let random variable $K_{w}$ be the number of times an entry is picked in $W$ steps.

$$
\begin{aligned}
\operatorname{Mean}\left(K_{w}\right) & =\sum_{i}^{W} E\left(I_{i}\right) \\
& =\sum_{i}^{W}\left(1 * \frac{k}{m}\right) \\
& =\frac{W * k}{m}
\end{aligned}
$$


We prove the theorem.

It can be concluded, from Theorem 1, that the expected number of times a entry is picked within $W$ steps is $\frac{W * k}{m}$. Thus each counter is reduced by $\frac{W * k}{m}$ within $W$ steps on average. If the maximum value $C$ is set to the average $\frac{W * k}{m}$, and after that no elements are mapped to the entry in the following $W$ steps, the counter is decreased to 0 with high probability. Therefore, we have the following corollary:

Corollary 1. The maximum value of a counter in CVS, denoted as $C$, is set to $\frac{W * k}{m}$.

\subsection{Error Analysis}

By cardinality estimate Equation 1, our algorithm has two sources of errors. The first one is the CVS size $m$. In fact the inaccuracy is caused by the bitmap sketch on which the CVS is based. An appropriate size $m$ can be determined such that the estimate error caused by the first source is less than a preset error threshold. More details can be found in [16]. In this paper, we focus on analyzing the second error source.

The second error source is the deviation of $z$ from its exact value, where $z$ is the number of zeroes in CVS. In essence, the deviation is caused by the random update mechanism. By Theorem 1, the number of times that a entry is picked within $W$ iterations is $C$ only on average. It is the statistic bias that gives rise to the deviation of $z$ from its exact value, which finally causes inaccuracy in cardinality estimation.

In the rest of this section, we will analyze the influence of the random update mechanism on the number of zeros in CVS. The following theoretical analysis will show that the inaccuracy caused by the random update mechanism can be very small, if the number of randomly chosen counters is set properly.

As shown in Fig. 2, there are two types of false states, which may cause the deviation of the number of zeros, namely False Empty (FE) and False NonEmpty (FNE). Suppose that the entry $i$ is set to $C$ at $t_{0}$ and no elements are 
mapped to the entry within the interval $\left[t_{0}, t_{1}\right]\left(t_{1}>t_{0}\right)$. (a) The entry $i$ is in False Empty at $t_{1}$ if $V[i]=0$ and $t_{1}<t_{0}+W$, as shown in Fig. 2(a). (b) The entry $i$ is in False non-Empty at $t_{1}$ if $V[i] \geq 1$ and $t_{1}>t_{0}+W$, as shown in Fig. 2(b)

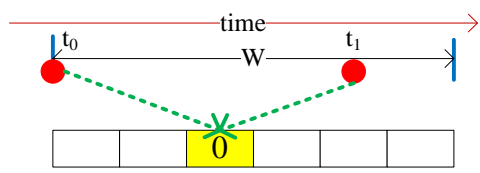

(a) False Empty

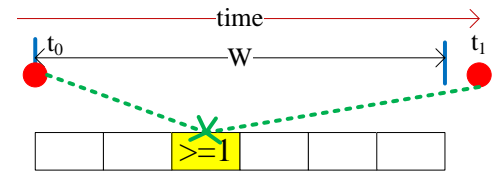

(b) False Non-Empty

Figure 2: Two cases in False State: (a)the entry in False Empty; (b) the entry in False Non-Empty.

For description convenience, we first introduce some notations. Let $\Delta t=$ $t_{1}-t_{0}$ denote the number of steps within which no elements are mapped to the entry. Let random variable $K_{i}$ be the number of times that the entry $i$ is chosen to decrement within $\Delta t$.

The probability that the entry is in FE can be derived as follows:

$$
\begin{aligned}
\operatorname{Pr}(F E) & =P_{r}(V[i]=0 ; \Delta t<W) \\
& =\sum_{i=C}^{W-1}(\operatorname{Pr}(V[i]=0 / \Delta t=i) * \operatorname{Pr}(\Delta t=i)) \\
& =\sum_{i=C}^{W-1}\left(\operatorname{Pr}\left(K_{i} \geq C / \Delta t=i\right) * \operatorname{Pr}(\Delta t=i)\right)
\end{aligned}
$$

where $K_{i}$ follows a Binomial distribution $B(\Delta t, k / m), \operatorname{Pr}(\Delta t=i)=(1-$ $k / m)^{i} *(k / m)$.

The random variable $\bar{A}$ denotes the event that no elements are mapped to the entry $i$ during $W$ iterations. Therefore, type 2 error rate is:

$$
\begin{aligned}
\operatorname{Pr}(F N E) & =\operatorname{Pr}(V[i]>0 ; \bar{A}) \\
& =\operatorname{Pr}(V[i]>0 / \bar{A}) * \operatorname{Pr}(\bar{A}) \\
& =\operatorname{Pr}\left(K_{i}<C / \bar{A}\right) * \operatorname{Pr}(\bar{A})
\end{aligned}
$$

where $K_{i}$ follows a Binomial distribution $B(W, k / m), \operatorname{Pr}\left(\bar{A}=(1-k / m)^{W}\right.$. 
From equations 3 , 4 , we obtain the probability that the entry state is false:

$$
\begin{aligned}
\operatorname{Pr}(\text { False_Rate })= & \operatorname{Pr}(F E)+\operatorname{Pr}(F N E) \\
= & \sum_{i=C}^{W-1}\left(\operatorname{Pr}\left(K_{i} \geq C / \Delta t=i\right) * \operatorname{Pr}(\Delta t=i)\right) \\
& +\operatorname{Pr}\left(K_{i}<C / \bar{A}\right) * \operatorname{Pr}(\bar{A})
\end{aligned}
$$

where $K_{i}$ follows Binomial distribution with parameter $N=\Delta t$ and $p=k / m$, $\operatorname{Pr}(\Delta t=i)$ and $\operatorname{Pr}(\bar{A})$ are related to the gap distribution that the entry is set.

To obtain $\operatorname{Pr}(\Delta t=i)$, we need to know the probability that the entry $i$ is set in a step, denoted as $p_{\text {set }}^{i}$. We assume that the underlying distribution of the input data $S$ dose not change with time. If the elements in $S$ follows uniform distribution, then $p_{\text {set }}^{i}=1 / m$. Otherwise, we just assume $1 / m$ to be the average of $p_{s e t}^{i}$ in practice. Hence, in this paper, we set $\operatorname{Pr}(\Delta t=i)=(1-1 / m)^{i} * 1 / m$ and $\operatorname{Pr}(\bar{A})=(1-1 / m)^{W}$.

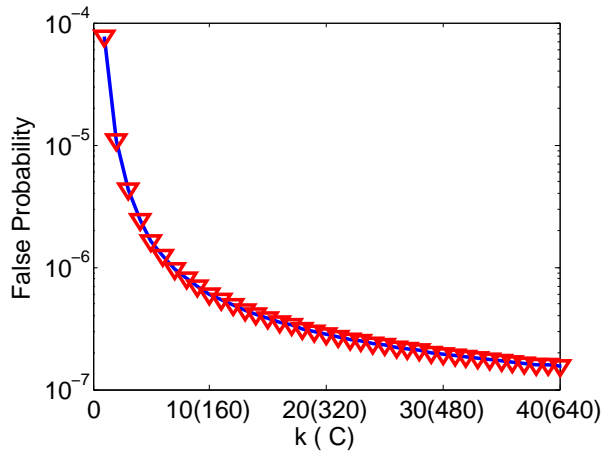

(a)

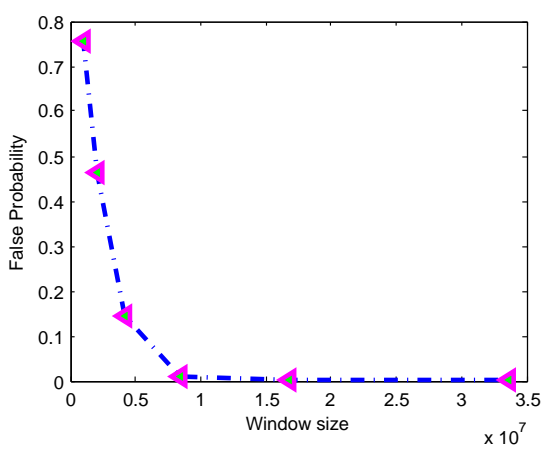

(b)

Figure 3: False probability with (a) different $k(C)$ and (b) with different window size $W$

Based Equation 5, for a given $W=2^{20}$ and $m=2^{16}$, Fig. 3(a) gives the false state rate with different $k$. It can be seen that the error rate decreases exponentially as $k$ increases, or equivalently increasing $C$. This indicates that a small $k$ is enough to reach a very small false state rate, i.e., only very few entries are needed to be picked in a step. Therefore, our algorithm is very fast but attains almost the same accuracy as bitmap sketch. 
If $k$ and $m$ (i.e., fixed $p$ ) is fixed, Fig. 3(b) presents the false state rate with different sliding window size $W$. It can be found that the false state rate is exponentially decreasing as $W$ increases. This can also be confirmed from another aspect. The rise of $W$ means that the total number of random selections during $W$ steps increases proportionally. Consequently, the expected number of times an entry is picked converges to its average, $C$, by the Law of Large Number. In the end, more accurate results are obtained as $W$ increases.

\subsection{Tradeoff Between Accuracy, Memory and Time Complexity}

As discussed in last section, the increase of $k$ can reduce the estimate error of CVS. However, the rise of $k$ means that the running time grows proportionally. Additionally, the memory requirement also increases with $\mathrm{k}$ because the parameter $C$ is positively proportional to $k$ by Equation 2. Thus, one key problem we have to address is to make a fine tradeoff between estimate error, memory and time complexity.

Estimate Error. In last section, the estimate error has been discussed in detail. To set $k$, keeping parameters $W$ and $m$ fixed, we can try different values of $k$ to find the least $k$ for a user-specified accuracy. In practical applications, the parameter $k$ may be very small. Usually, an integer less than 20 is sufficient, as is shown in Fig. 3(a),

Space Complexity. In this paper, the space complexity is measured by the number of bits. The amount of memory space cost by our algorithm is $C * m$ where $m$ is the CVS size. For a given data set, suppose that the maximum cardinality of the set is known, the appropriate size $m$ for a specified accuracy can be obtained according to [16]. For a given $W$ and $k$, the maximum value $C$ of a counter is a constant. As a result the memory space is determined only by the number of entries $m$.

Time Complexity. For each element $e \in S$, our algorithm firstly needs to perform mapping operation $h(e)$ and set the entry $V[h(e)]$ to $C$. After the mapping operation, it picks $k$ entries, each of which is decremented by 1 . Because the mapping operation only uses constant time, the time cost of our algorithm is 
dominated only by $k$. Therefore, we obtain the following result: For a constant $k$, our algorithm takes constant time, $O(1)$ running time, independent of the CVS size $m$, sliding window size $W$ and the data stream $S$.

It is clear, from the analysis above, that the parameter $k$ is of great importance to our algorithm performance. Keep parameters $W$ and $m$ fixed, and try different $k$ using Equation 5 . The least $k$ is chosen such that the estimate error is less than a user-specified threshold.

\section{Experiments}

In this section, the performance evaluation of our proposed CVS will be carried out using four data sets. All algorithms are implemented in Java. All the experiments are performed on a computer with a 4 -Core $2.67 \mathrm{GHz}$ Intel $\mathrm{CPU}$ and 4GB main memory. In order to obtain the average accuracy and running time, 10 trials of experiments are carried out for each case. Results show that our CVS schema achieves high accuracy and runs faster than TSV and the indexed sketch.

\subsection{Data sets}

Our experiments are performed on both artificial and real-life data sets. Details of used data set are presented in Table 3. Below, we briefly describe each data set.

Table 3: Details of data sets used in our experiments

\begin{tabular}{llcc}
\hline Data set & Cardinality $(\mathrm{max} / \mathrm{min})$ & \# items & Fluctuation \\
\hline DS1 & $9.5 \mathrm{e} 3 / 800\left(\right.$ on $\left.W=2^{14}\right)$ & $6 \mathrm{e} 5$ & sudden \\
DS2 & $3.4 \mathrm{e} 4 / 2.5 \mathrm{e} 4\left(\right.$ on $\left.W=2^{20}\right)$ & $2.5 \mathrm{e} 7$ & moderate \\
DS3 & $8 \mathrm{e} 3 / 2 \mathrm{e} 3\left(\right.$ on $\left.W=2^{16}\right)$ & $7.7 \mathrm{e} 7$ & moderate \\
DS4 & $1.7 \mathrm{e} 4 / 8 \mathrm{e} 3\left(\right.$ on $\left.W=2^{19}\right)$ & $4 \mathrm{e} 7$ & sudden \\
\hline
\end{tabular}

The first data set is a artificial data set (DS1) generated by a pseudo random number generator. It consists of $6 * 10^{5}$ elements. There exist sharp changes in cardinality within a short time, as shown in Fig. 5 . 
The second (DS2) is a real-world IP traffic trace collected from Internet backbone links on the 1 th of January 2010 [28, which consists of about $4 * 10^{7}$ IP packets headers (about 1.5GB compressed data). The cardinality of the data set is defined as the number of distinct (destination, source)-IP pairs.

The third (DS3) is the 1998 World Cup Web access logs [29], consisting of 70-day user request records. We extract the first 40 day's access logs (over 7.7e7 records). In our experiments, we exploit the data set to track the number of distinct requests.

The fourth (DS4) is a DDoS network trace [30] file collected from Internet backbone links (about 1GB compressed data). Similar to DS2, each record consists of a pair of source and destination IP. When DDoS attacks breaks out, the cardinality of the data stream will fall drastically. Thus, the data set contains sudden cardinality changes, as shown in Fig. 8

\subsection{Evaluation Criteria}

As mentioned in Section 3.4. CVS algorithm has two error sources. In this paper we only focus on errors introduced by random update mechanism. To eliminate the effect of the first factor, we compare the results of CVS algorithm with that of TSV (Note that TSV has the same accuracy as bitmap sketch). Experiments show that CVS can achieve almost the same accuracy as bitmap sketch.

To evaluate the estimate errors introduced by random update mechanism, we employ three widely used criteria: Relative Deviation (ReDev), Bias and Standard Deviation (stdDev). The three metrics are formally defined as follows: Let $\hat{n}_{i}, n_{i}$ be two estimates generated respectively by CVS and TSV at time $i$.

- ReDev: $r_{i}=\left(n_{i}-\hat{n}_{i}\right) / n_{i}$, which indicates the deviation degree from $n_{i}$ at time $t$.

- Bias: Bias $=\operatorname{Mean}(R)$, where $R=\left\{r_{1}, r_{2} \ldots r_{i} \ldots\right\}$. The Bias metric equals to the Mean of relative error sequence. 
- stdDev: $s t d D e v=S t d(R)$. The stdDev metric equals to the Variance of $R$.

Table 4: Parameter settings for experiments on four data sets
\begin{tabular}{llccc} 
Parameter & DS1 & DS2 & DS3 & DS4 \\
\hline CVS size $m$ & $2^{13}$ & $2^{16}$ & $2^{13}$ & $2^{15}$ \\
Window size $W$ & $2^{14}$ & $2^{20}$ & $2^{16}$ & $2^{19}$ \\
\hline
\end{tabular}

\subsection{Experimental Results}

Our experiments are carried out on the artificial data set, and then on the three real-world data sets. Details of parameter settings for CVS size $m$ and sliding window size $W$ can be found in Table 4 . Note that we select the size $m$ so that the stdDev of TSV is less or equal than 0.01 according to paper [16].

\begin{tabular}{|c|c|c|}
\hline$k$ & stdBias & stdDev \\
\hline 5 & 0.0463 & 0.0168 \\
\hline 10 & 0.0239 & 0.0101 \\
\hline 20 & 0.0122 & 0.0075 \\
\hline 40 & 0.0061 & 0.0049 \\
\hline 60 & 0.0042 & 0.0045 \\
\hline 80 & 0.0031 & 0.0039 \\
\hline 100 & 0.0025 & 0.0036 \\
\hline 120 & 0.0023 & 0.0033 \\
\hline 150 & 0.0017 & 0.0031 \\
\hline
\end{tabular}

\subsubsection{Results on artificial data set}

For varied $k$, the CVS and TSV are carried out on DS1 with the fixed $W$ and $m$. Based on their respective estimates, three types of estimate errors mentioned above, i.e. ReDev, Bias and stdDev, are calculated. The ReDev of CVS is shown in Fig. 4(a) Details of Bias and stdDev are presented in Table 5. a schematic view of the table is given in Fig. 4(b). From these results, we obtain the following conclusions. 


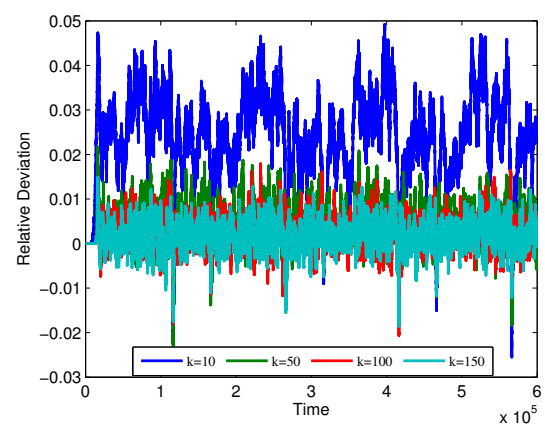

(a) ReDev

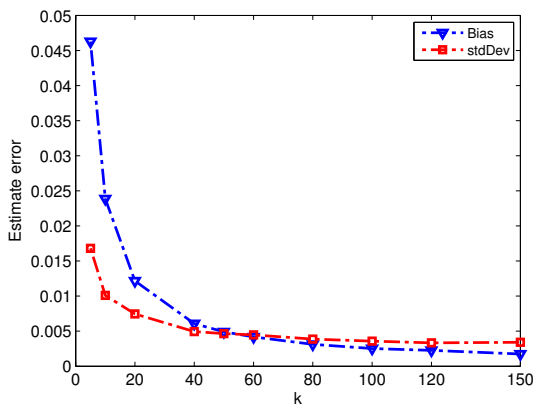

(b) A schematic view of Bias and stdDev

Figure 4: Estimate errors of CVS on DS1

Firstly, the error introduced by random update is very small. When $k$ is assigned to a small value 2 , the relative deviations range from -0.02 to 0.05 (Fig. 4(a), at most 5\% away from TSV. The bias and stdDev are 0.046, 0.0168 respectively (Table 5). However, when $k=50$, the vast majority of relative deviations are less than 0.01; the average error (Bias) is 0.0049 , only $\% 0.49$ away from TSV.

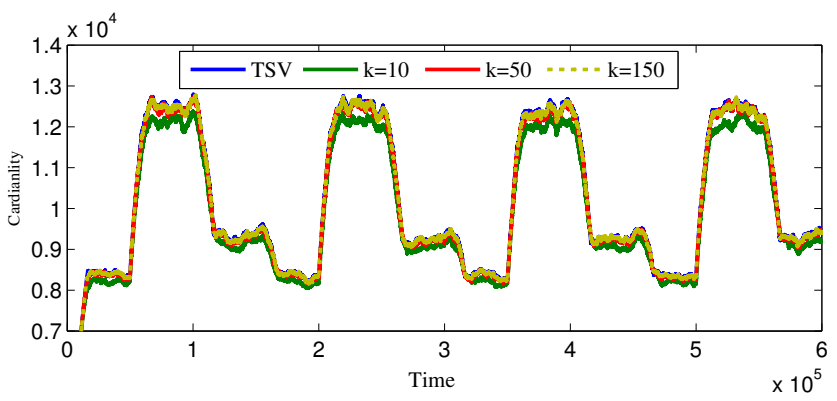

Figure 5: Estimates on DS1 for different $k$

Secondly, the estimation errors fall with the increase of parameter $k$. Take the Bias and stdDev for example. As shown in Fig. 4(b), Bias and standard deviation fall almost exponentially with $k$ growing. This is consistent with our error analysis in Section 3.4 .

Lastly, sharp fluctuations of cardinality have little effects on the approxima- 
tion accuracy. The cardinality estimates of CVS on DS1 with $k=10,50,150$ are shown in Fig. 5. By comparison with TSV, it can be seen that abrupt cardinality changes in DS1 have little impact on the accuracy of estimates. We will further study the problem on a real-life data set DS4.

\subsubsection{Results on real-world Data sets}

Table 6: Bias and stdDev on DS2, DS3 and DS4

\begin{tabular}{|c|c|c|c|c|c|c|}
\hline \multirow[b]{2}{*}{$k$} & \multicolumn{2}{|c|}{ DS2 } & \multicolumn{2}{|c|}{ DS3 } & \multicolumn{2}{|c|}{ DS4 } \\
\hline & Bias & stdDev & Bias & stdDev & Bias & stdDev \\
\hline$\overline{2}$ & 0.0310 & 0.0072 & 0.0039 & 0.0090 & 0.0075 & 0.0156 \\
\hline 5 & 0.0165 & 0.0056 & 0.0016 & 0.0063 & 0.0030 & 0.0087 \\
\hline 10 & 0.0033 & 0.0034 & $8.0 \mathrm{e}-4$ & 0.0051 & 0.0014 & 0.0061 \\
\hline 20 & 0.0016 & 0.0028 & $4.0 \mathrm{e}-4$ & 0.0038 & $7.1 \mathrm{e}-4$ & 0.0044 \\
\hline 30 & 0.0011 & 0.0025 & $2.7 \mathrm{e}-4$ & 0.0034 & $4.7 \mathrm{e}-4$ & 0.0037 \\
\hline 40 & $7.9 \mathrm{e}-4$ & 0.0023 & $2.1 \mathrm{e}-4$ & 0.0031 & $3.8 \mathrm{e}-4$ & 0.0033 \\
\hline 50 & $6.6 \mathrm{e}-4$ & 0.0022 & $1.7 \mathrm{e}-4$ & 0.0029 & $2.3 \mathrm{e}-4$ & 0.0028 \\
\hline
\end{tabular}

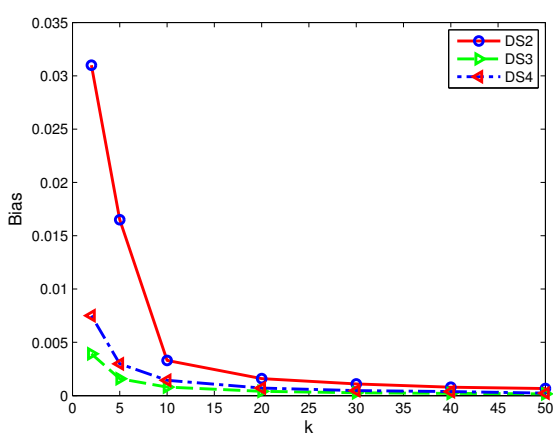

(a) Bias on three data sets

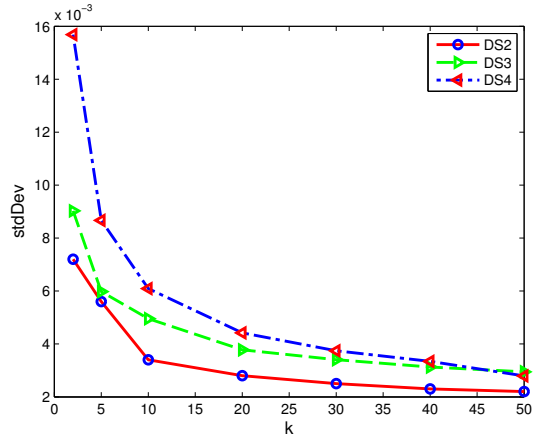

(b) stdDev on three data sets

Figure 6: Two schematic pictures of (a) Bias and (b) stdDev with different $k$

In last section, an artificial data set has been used to evaluate the accuracy of our proposed CVS. In this section, the accuracy of CVS will be further tested against three real-world data sets (DS2, DS3 and DS4). Besides, we will study the effects of sudden changes on the estimate accuracy in detail. For each $k$ (2, $5,10,20,30,40,50)$, the ReDev, Bias and StdDev of CVS on each data set are obtained. Details of Bias and stdDev are presented in Table 6 a schematic view of the table is given in Fig. 6. The ReDev is shown in Fig. 7. 

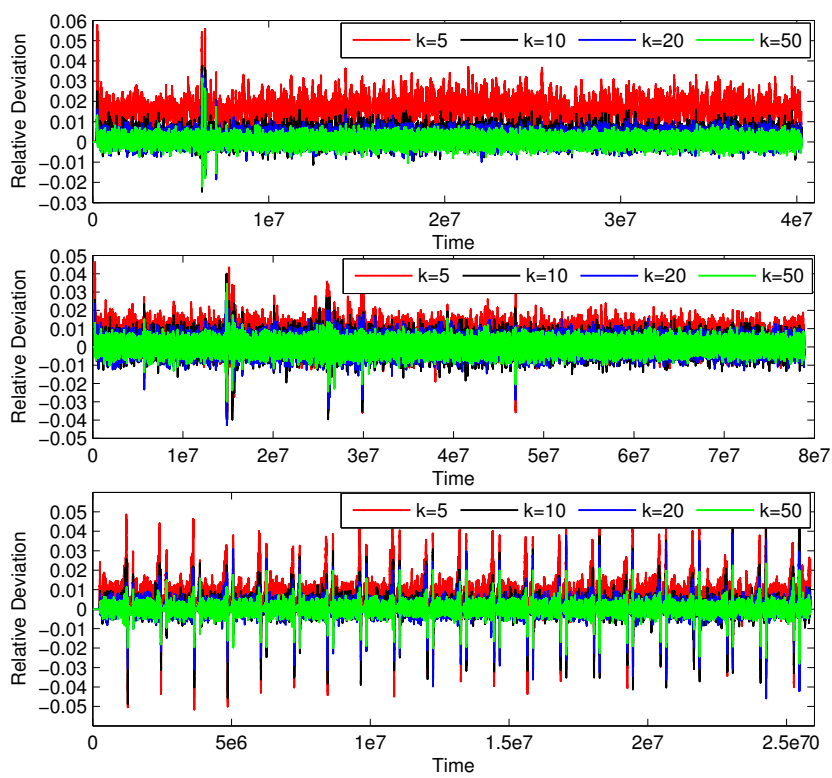

Figure 7: ReDev on DS2 (top), DS3 (middle)and DS4 (bottom)

Relative Deviation. Fig. 7 shows ReDev of CVS on the three data sets respectively. Similar to ReDev on the synthetic data set DS1, CVS yields a little more deviation when pick times $k$ is small (for $k=5$, the ReDev varies from -0.06 to 0.06 , about $6 \%$ of deviation from TSV). However, the error drops rapidly as $k$ rises. When $k=10$, CVS offers much higher accuracy than when $k=5$. Most of ReDev ranges from -0.01 to 0.01 , only $\% 1$ away from TSV. Fig. 7 shows that there are some peaks in relative deviation on DS4 when $k<10$. This issue will be further discussed later.

Bias. Fig. 6(a) plots the Bias changes on three data sets with $k$; its details are listed in Table 6. When $k$ is small (less than 10), the Biases on three data sets vary in a relatively wide range, from 0.03 to 0.005 . However, the Bias tends to converge as $k$ increases. Furthermore, as shown in Fig. 6(a) the Bias decreases almost exponentially with $k$ increasing.

Standard Deviation. Fig. 6(b) presents the variation of stdDev with different $k$; its details are also listed in Table 6. The stdDev of CVS has similar 
change tendency: it rapidly decreases with $k$ increase.
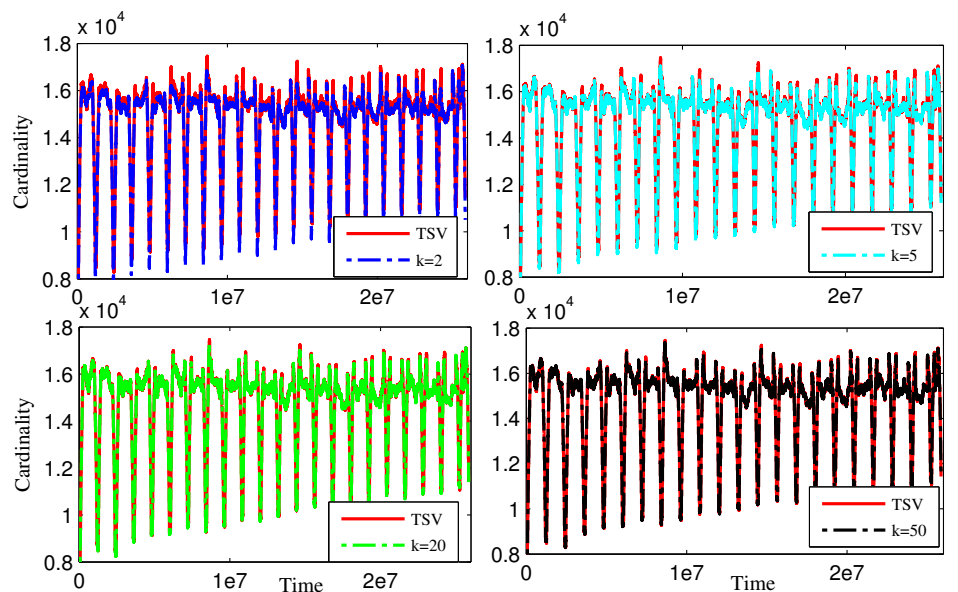

Figure 8: Estimate comparisons between TSV and CVS with $k=2$ (top-left), $k=5$ (topright), $k=20$ (bottom-left) and $k=50$ (bottom-right)
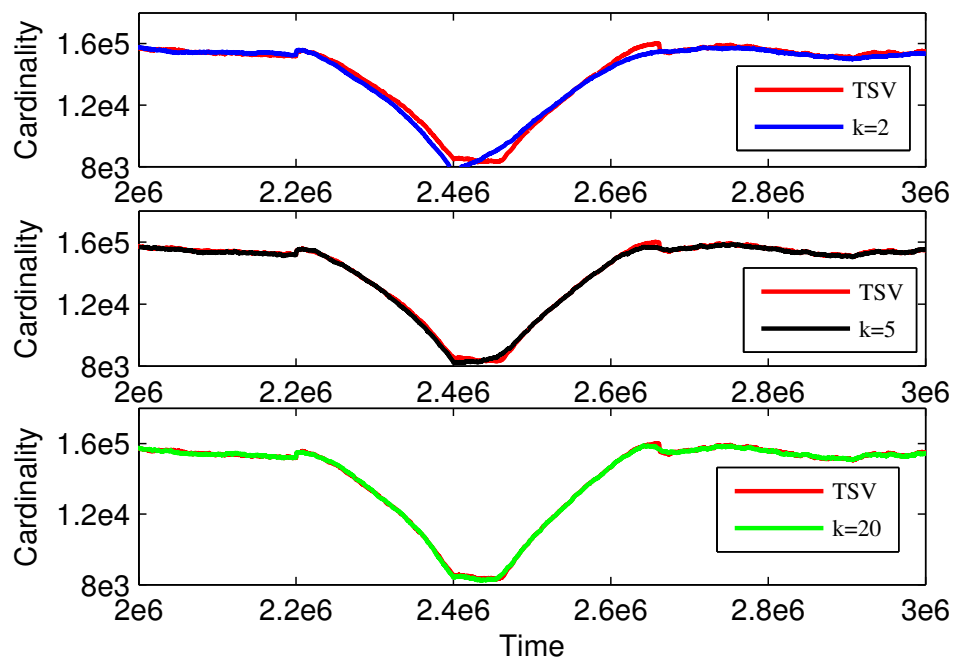

Figure 9: A slice of Fig. 8 around an abrupt change-point: $k=2$ (top), $k=5$ (middle) and $k=20$ (bottom)

Influence of abrupt cardinality changes. Here we focus on the influence 
of the abrupt cardinality changes on the estimate accuracy. As mentioned above, the data set DS4 includes DDoS attacks, which may give rise to a sharply decrease within a short time, as shown in Fig. 8.

Fig. 8 compares estimates of CVS with ones of TSV. For clear illustration, we extract a slice of Fig. 8 around a change point, shown in Fig. 9 . Fig. 9 shows that CVS incurs a series of obvious deviations around the sudden change point when $k$ is small. These deviation almost disappears as $k$ increases. The reasons for this phenomenon are that: 1) the actual number of times a counter is updated is apt to deviate from the average number $C$ when $k$ is small; but

2 ) it tends to converge to $C$ as $k$ increases, as is shown in Fig. 9 (middle and bottom).

From the analysis above, we found that only a small $k$ is needed for attaining a small deviation (e.g, less than 0.01). This means that CVS just randomly picks a small portion of counters in one step, which requires constant time for a small fixed $k$. This serves as a foundation for applying CVS in online scenarios. On the other hand, the error changes in Fig. 6(b) 6(a) are consistent with the False State Probability in Fig. 3(a), This demonstrates our theoretical error analysis of CVS from another aspect.

\subsection{Comparison}

This section compares three methods: CVS, TSV and auxiliary indexing method in terms of time, space and accuracy. The comparison results are presented in Fig. 7. TSV can be considered as a special case of CVS, where $C=W$ and all non-empty entries are decremented by 1 in each step. So it cost $O(m)$ time in each step and $m * \log W$ memory space. TSV has no loss of accuracy compared with bitmap sketch. The main limitation of TSV is that it cost too much update time in large-scale real-time data streams.

Table 7: Comparison with TSV and auxiliary
\begin{tabular}{llll} 
Method & Time & Space (bit) & Accuracy \\
\hline TSV & $O(m)$ & $m * \log W$ & $=$ D-Bitmap \\
Auxiliary Indexing & $O(\log m)$ & $O(m * \log m)$ & $=$ D-Bitmap \\
CVS & $O(1)$ & $m * \log C$ & $\leq$ D-Bitmap \\
\hline
\end{tabular}


Auxiliary indexing method maintains a temporally sorted list to facilitate evicting process. It costs $O(m * \log m)$ memory space. In each step, it needs to scan a number of entries for evicting stale information, $\operatorname{costing} O(\log m)$ time on average. It has the same accuracy as bitmap sketch. Its disadvantage is that it consumes too large memory space in large-scale data streams.

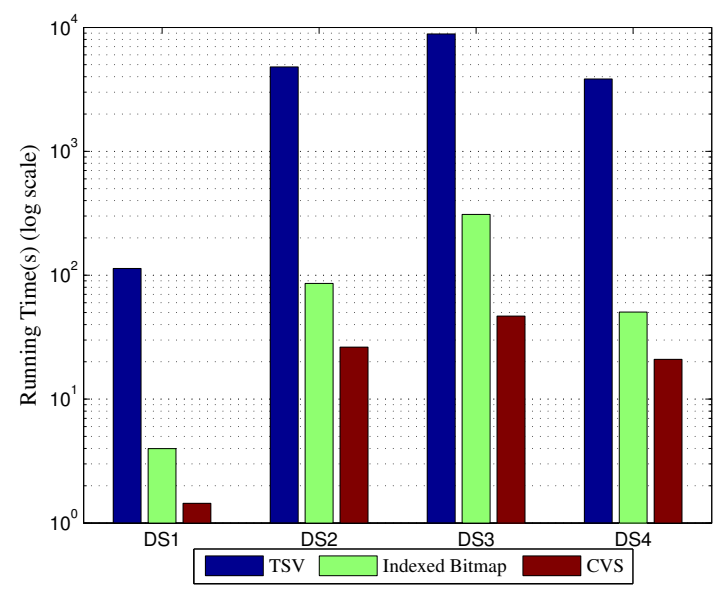

Figure 10: Running time comparison on four data sets

The three algorithms are carried out on the four data sets and their running time is measured respectively, shown in Fig. 10. The running time of CVS is about two orders of magnitude smaller than that of TSV, and is at most 1/2 of that of the auxiliary indexing method.

CVS only needs constant running time in each step, and $m * \log C$ memory space $(C \ll W)$, although it has a very small loss of accuracy compared with TSV and indexing method. The loss of accuracy can be reduced to a very small level by tuning the parameter $k$ (the number of entries picked in a step).

\section{Conclusions}

In this paper, we present a counter vector sketch data structure, which requires $O(1)$ update time using a random update mechanism, and consumes much smaller memory space schema compared with TSV [21] and the auxiliary 
indexing method 22]. Base on the CVS data structure, a streaming algorithm is designed for timely estimating the cardinality of large-scale high-speed data streams over sliding windows.

The key idea behind our solution is to use a random update method for timely evicting stale information. The correctness of the method is guaranteed by Large Number Law in Probability Theory. The idea proposed in the paper can also be used to extend other synopsis data structures to the sliding window model, such as multiple resolution bitmap [31, self-learning bitmap [32], although our work is discussed only on the basis of direct bitmap sketch.

In addition, our schema can be easily extended to distributed scenarios. Assuming that $n$ CVSes the same size are deployed at $n$ workstations, respectively. When a center initiates a query, each workstation sends the bitmap corresponding to its CVS (non-empty entries set to 1 , otherwise 0 ). Thus, we can obtain the overall bitmap sketch by applying logical $O R$ operation to all of bitmaps bit by bit.

In many real-life applications, data streams are often accompanied by concept drift, where the underlying distribution of samples varies significantly over time. In our work, the changing distribution may give rise to the drastic fluctuations of cardinality, which may result in the obvious estimate errors. Inspired by B. Krawczyk and M. Wozniak's work [8, 9, 10, in future, we plan to 1) extend our method to an adaptive-window-size model to cope with data streams with

concept drift, 2) design a time sliding-window-based CVS so that it can work with data streams with bursty, and 3) incorporate the CVS into a classifier.

\section{Acknowledgment}

This work is supported under the National High Technology Research and Development Program of China grants 2012AA01A510 and 2012AA01A509, and partially supported under the National Science Foundation of China (NSFC) grant 61402518 and the Jiangsu Province Science Foundation for Youths grant BK20150722. 


\section{References}

[1] B. Krawczyk, J. Stefanowski, M. Wozniak, Data stream classification and big data analytics, Neurocomputing 150 (2015) 238-239.

[2] S. Muthukrishnan, Data streams: Algorithms and applications, Foundations and Trends in Theoretical Computer Science 1 (2) (2005) 1-39. doi:10.1561/0400000002.

[3] B. Babcock, S. Babu, M. Datar, R. Motwani, J. Widom, Models and issues in data stream systems, in: Proceedings of the Twenty-first ACM SIGACTSIGMOD-SIGART Symposium on Principles of Database Systems, 2002, pp. 1-16. doi:10.1145/543613.543615

[4] N. Wang, M. J. Er, M. Han, Parsimonious extreme learning machine using recursive orthogonal least squares, IEEE Trans. Neural Netw. Learning Syst. 25 (10) (2014) 1828-1841. doi:10.1109/TNNLS.2013.2296048.

[5] N. Wang, M. Han, N. Dong, M. J. Er, Constructive multi-output extreme learning machine with application to large tanker motion dynamics identification, Neurocomputing 128 (2014) 59-72. doi:10.1016/j.neucom.2013. 01.062 .

[6] N. Wang, M. J. Er, X. Meng, X. Li, An online self-organizing scheme for parsimonious and accurate fuzzy neural networks, Int. J. Neural Syst. 20 (5) (2010) 389-403. doi:10.1142/S0129065710002486.

[7] N. Wang, M. J. Er, M. Han, Generalized single-hidden layer feedforward networks for regression problems, IEEE Trans. Neural Netw. Learning Syst. 26 (6) (2015) 1161-1176. doi:10.1109/TNNLS.2014.2334366

[8] B. Krawczyk, M. Wozniak, Incremental weighted one-class classifier for mining stationary data streams, J. Comput. Science 9 (2015) 19-25. doi: $10.1016 / \mathrm{j} \cdot$ jocs.2015.04.024. 
[9] B. Krawczyk, M. Wozniak, One-class classifiers with incremental learning and forgetting for data streams with concept drift, Soft Comput. 19 (12) (2015) 3387-3400. doi:10.1007/s00500-014-1492-5.

[10] B. Krawczyk, M. Wozniak, Reacting to different types of concept drift with adaptive and incremental one-class classifiers, in: 2nd IEEE International Conference on Cybernetics, 2015, pp. 30-35. doi:10.1109/CYBConf .2015. 7175902

[11] C. Estan, G. Varghese, New directions in traffic measurement and accounting: Focusing on the elephants, ignoring the mice, ACM Trans. Comput. Syst. 21 (3) (2003) 270-313. doi:10.1145/859716.859719

[12] J. Cao, Y. Jin, A. Chen, T. Bu, Z. Zhang, Identifying high cardinality internet hosts, in: 28th IEEE International Conference on Computer Communications INFOCOM, 2009, pp. 810-818. doi:10.1109/INFCOM.2009. 5061990 .

[13] W. Chen, Y. Liu, Y. Guan, Cardinality change-based early detection of large-scale cyber-attacks, in: Proceedings of the IEEE INFOCOM 2013, Turin, Italy, April 14-19, 2013, 2013, pp. 1788-1796. doi : 10.1109/INFCOM. 2013.6566977.

[14] P. B. Gibbons, Y. Matias, Synopsis data structures for massive data sets, in: Proceedings of the Tenth Annual ACM-SIAM Symposium on Discrete Algorithms, 17-19 January 1999, Baltimore, Maryland., 1999, pp. 909-910.

[15] P. Flajolet, G. N. Martin, Probabilistic counting algorithms for data base applications, J. Comput. Syst. Sci. 31 (2) (1985) 182-209. doi:10.1016/ 0022-0000(85) 90041-8.

[16] K. Whang, B. T. V. Zanden, H. M. Taylor, A linear-time probabilistic counting algorithm for database applications, ACM Trans. Database Syst. 15 (2) (1990) 208-229. doi:10.1145/78922.78925. 
[17] F. Giroire, Order statistics and estimating cardinalities of massive data sets, Discrete Applied Mathematics 157 (2) (2009) 406-427. doi:10.1016/j. dam.2008.06.020.

[18] M. Durand, P. Flajolet, Loglog counting of large cardinalities (extended abstract), in: 11th Annual European Symposium, 2003, pp. 605-617. doi: 10.1007/978-3-540-39658-1_55.

[19] B. H. Bloom, Space/time trade-offs in hash coding with allowable errors, Commun. ACM 13 (7) (1970) 422-426. doi:10.1145/362686.362692.

[20] M. Datar, A. Gionis, P. Indyk, R. Motwani, Maintaining stream statistics over sliding windows, SIAM J. Comput. 31 (6) (2002) 1794-1813. doi: $10.1137 /$ S0097539701398363.

[21] H. Kim, D. R. O'Hallaron, Counting network flows in real time, in: Proceedings of the Global Telecommunications Conference, 2003, pp. 38883893. doi:10.1109/GLOCOM.2003.1258959.

[22] É. Fusy, F. Giroire, Estimating the number of active flows in a data stream over a sliding window, in: Proceedings of the Fourth Workshop on Analytic Algorithmics and Combinatorics, 2007, pp. 223-231. doi: $10.1137 / 1.9781611972979 .9$

[23] Y. Chabchoub, G. Hébrail, Sliding hyperloglog: Estimating cardinality in a data stream over a sliding window, in: The 10th IEEE International Conference on Data Mining Workshops, 2010, pp. 1297-1303. doi:10. 1109/ICDMW. 2010.18

[24] A. Metwally, D. Agrawal, A. El Abbadi, Why go logarithmic if we can go linear?: Towards effective distinct counting of search traffic, in: 11th International Conference on Extending Database Technology, 2008, pp. 618-629. doi:10.1145/1353343.1353418.

[25] K. Aouiche, D. Lemire, A comparison of five probabilistic view-size estimation techniques in OLAP, CoRR abs/cs/0703058. 
[26] K. Mouratidis, D. Papadias, Continuous nearest neighbor queries over sliding windows, IEEE Tran. on Know. and Data Eng. 19 (6) (2007) 109-120. doi:10.1109/TKDE.2007.1020.

[27] F. Deng, D. Rafiei, Approximately detecting duplicates for streaming data using stable bloom filters, in: Proceedings of the ACM SIGMOD International Conference on Management of Data, 2006, pp. 25-36. doi: 10.1145/1142473.1142477.

[28] Waikato Internet Traffic Storage project, http://wand.net.nz/wits/ index.php (2010).

[29] M. Arlitt, T. Jin, 1998 world cup web site access logs, http://www.acm. org/sigcomm/ITA/ (1998).

[30] The CAIDA UCSD DDoS Attack 2007 Dataset, http://www.caida.org/ data/passive/ddos-20070804_dataset.xml (2007).

[31] C. Estan, G. Varghese, M. E. Fisk, Bitmap algorithms for counting active flows on high-speed links, IEEE/ACM Trans. Netw. 14 (5) (2006) 925-937. doi:10.1145/1217709

[32] A. Chen, J. Cao, Distinct counting with a self-learning bitmap, in: Proceedings of the 25th International Conference on Data Engineering, 2009, pp. 1171-1174. doi:10.1109/ICDE.2009.193. 


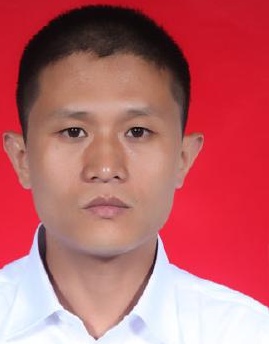




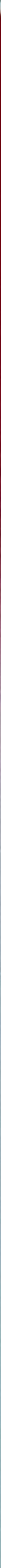




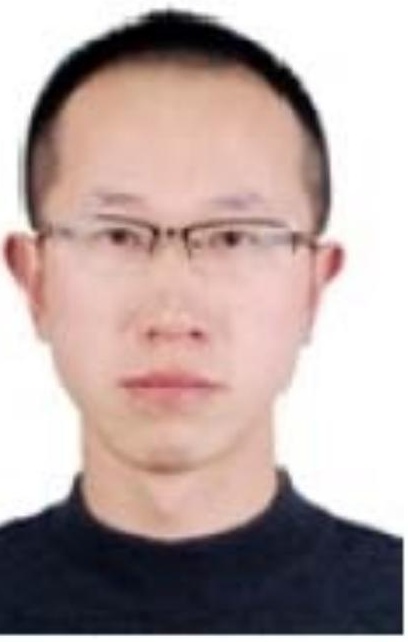




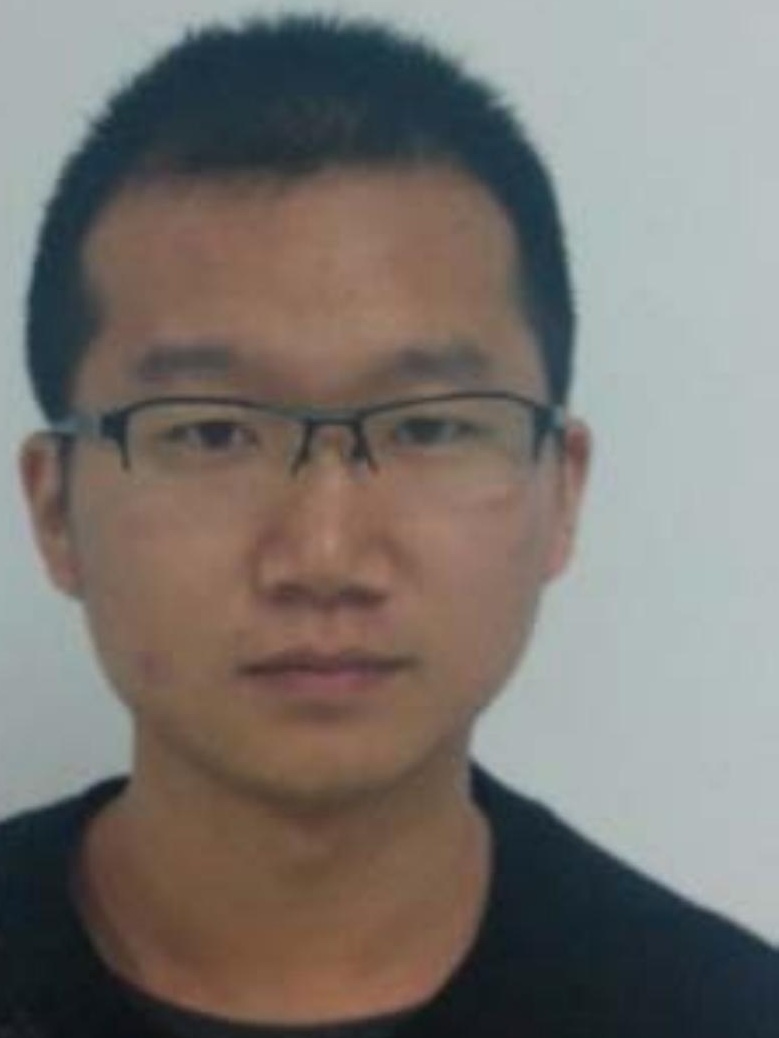

